\title{
A Novel Lenticular Arena to Quantify Locomotor Competence in Walking Fruit Flies
}

NALONG TOM MEKDARA ${ }^{1}$, JOY JUNE GOTO ${ }^{2}$, SONGITA CHOUDHURY ${ }^{3}$, PRASONG JERRY MEKDARA ${ }^{1}$, NICHOLAS YINGST ${ }^{3}$, YU CAO ${ }^{4}$, OTTO BERG ${ }^{2}$, AND ULRIKE KATHARINA MÜLLER ${ }^{1}$

\begin{abstract}
Drosophila melanogaster has become an important invertebrate model organism in biological and medical research, for mutational and genetic analysis, and in toxicological screening. Many screening assays have been developed that assess the flies' mortality, reproduction, development, morphology, or behavioral competence. In this study, we describe a new assay for locomotor competence. It comprises a circular walking arena with a lenticular floor and a flat cover (the slope of the floor increases gradually from the center to the edge of the arena) plus automated fly tracking and statistical analysis. This simple modification of a flat arena presents a graduated physical challenge, with which we can assess fine gradations of motor ability, since a fly's time average radial distance from the arena center is a direct indicator of its climbing ability. The time averaged distribution of flies as a function of slope, activity levels, and walking speed, yields a fine grained picture of locomotory ability and motivation levels. We demonstrate the strengths and weaknesses of this assay (compared with a conventional tap-down test) by observing flies treated with a neurotoxin (BMAA) that acts as a glutamate agonist. The assay proves well suited to detect dose effects and progression effects with higher statistical power than the traditional tap-down, but it has a higher detection limit, making it less sensitive to treatment effects.
\end{abstract}

Drosophila melanogaster has become an important invertebrate model organism for toxicological screens (Wolf et al., 2002; Peterson et al., 2008; Tiwari et al., 2011), genetic screening, and mutational analysis (e.g., Johnston, 2002; Giacomotto and Ségalat, 2010). Fruit flies are also an important model for neurodegenerative diseases (e.g., Bilen and Bonini, 2005; Lessing and Bonini, 2009; Pienaar et al., 2010) and a potential model for therapeutic drug

\footnotetext{
${ }^{1}$ Department of Biology, California State University, Fresno, California

${ }^{2}$ Department of Chemistry, California State University, Fresno, California

${ }^{3}$ Department of Electrical and Computer Engineering, California State University, Fresno, California

${ }^{4}$ Department of Computer Science and Engineering, University of Tennessee at Chattanooga, Chattanooga, Tennessee
} 
discovery (Pandey and Nichols, 2011).Many assays have been developed to quantify mortality, development, morphology, or behavioral competence (for a review see Rand, 2010).

With the advent of digital video and automated analysis, behavioral screening is reaching levels of sophistication sufficient to match the screening needs of mutational and toxicological research (e.g., Wehrhahn et al., '82; Valente et al., 2007; Katsov and Clandinin, 2008; Branson et al., 2009; Dankert et al., 2009). Yet automated image analysis also puts new constraints on experimental designs. Apart from imaging constraints (minimal shadows and highlights; evenly colored, matte backgrounds), many assays limit available space to prevent locomotory behaviors that confound automated analysis (Branson et al., 2009: prevent climbing vertical walls with thermal barriers; Simon and Dickinson, 2010: limit height to prevent flight). It is important to develop meaningful performance parameters that are not just easy to quantify automatically, but that also allow us to recognize increasingly subtle behavioral differences between flies.

\section{Behavioral Assays for Fruit Fly Locomotor Competence}

A popular behavioral assay for locomotor competence in Drosophila is the tap-down (e.g., Gargano et. al., 2005; Ruan and Wu, 2008; Zhou et al., 2010). Adult fruit flies are negatively geotactic and will climb up when shaken to the bottom of a vial. The tap-down tests locomotor competence at a single moment with a binary result, making this a simple assay, but also limiting its diagnostic value. To capture differences in motor ability with higher resolution, labs have developed more sophisticated versions by staggering tap-down events (Benzer, '67). There are other assays that focus on reactive behavior ("reactive" as described by Connolly, '67), such as Buridan's paradigm (visual stimulus; Götz, '80), a variation of Benzer's countercurrent fractionation device (combination of geotaxis and visual stimulus; Markow and Merriam, '77), and an air-puff arena (mechanical stimulus; Slawson at al., 2009). There are also assays that focus on spontaneous locomotory behavior, such as the bifurcating negative geotaxis assay (Hirsch, '59; Hirsch and Erlenmeyer- Kimling, '62; Hostetter and Hirsch, '67; Ricker and Hirsch, '88; Toma et al., 2002), square arenas (e.g., Martin et al., '99a), or circular arenas (e.g., LeBourg, '83; Branson et al., 2009; Simon and Dickinson, 2010). Reactivity assays measure the combined ability to walk and to respond to external stimuli ( = external motivation). Activity-based assays are more suitable to detect differences in internal motivation ( = activity levels). However, by adding internal motivation as a variable, assays measuring spontaneous behavior are also more likely to produce data with a larger variation.

As video technology and automated image analysis are becoming available, new assays have been developed (e.g., Hoy et al., '96; Martin 2004; Branson et al., 2009). There are several software options for automated video analysis that yield information about the behavior of fruit flies in a two-dimensional space (e.g., freeware: Ctrax developed at Caltech; commercial product: EthoVision developed by Noldus). Automated video analysis facilitates the analysis of long-term behavioral patterns and therefore can be used in assays that are not focused on 
reactive behavior (e.g., Fry et al., 2003; Martin, 2003; Martin, 2004; Valente et al., 2007; Grover et al., 2008; Dankert et al., 2009; Ristroph et al., 2009).

\section{Behavioral Assays Need Discriminating Performance Parameters}

Screening studies are often more discovery science than hypothesis driven. Scientific hypotheses and questions help scientists limit the type and quality of performance parameters to be measured, and these constraints then lead to specific experimental designs. Screening studies, on the other hand, benefit from quantifying as many parameters as possible, with constraints imposed mainly by manpower, statistical power, and data management. This breadth places a burden on screening assays (1) to identify parameters that can discriminate performance and (2) to elicit behaviors that facilitate discrimination. The tap-down for example uses a binary performance parameter (successfully passing a predefined height mark within a predefined time) in an assay that presents a physical challenge (vertical climb) to facilitate discrimination.

When using automated video analysis, obvious parameters to describe locomotory behavior are position, speed, and acceleration, both translational and angular. Yet these parameters might not be the most discriminating given the natural variation in spontaneous behaviors (such as chasing, courtship, or foraging). Many locomotory assays (e.g., tap-down) use reactive behavior to narrow the repertoire and eliminate internal motivation; they may limit gender to exclude certain types of behavior; and they may present a physical challenge, such as a vertical incline, or centrifuging (Ruan and Wu, 2008), to amplify performance differences. In general, performance parameters for screening assays must not only be easy to quantify, but they should also have a high signal-to-noise ratio. Hence, parameters should preferably use original or integrated position data (such as temporal and spatial averages) rather than spatial or temporal derivatives (such as speed or curvature) (Harper and Blake, '89; Walker, '98). They should also discriminate with sufficient statistical power. Hence, parameters should have low variation, they should be continuous rather than binary or ranked variables, and they should amplify differences between individuals and reduce variation within individuals.

Locomotory screening assays approach organisms as a black box, with locomotory behavior as the visible manifestation of whatever changes occurred inside the organism. Motor behavior is influenced by both locomotory motivation and motor ability. Assays that can discriminate between changes in motivation and ability have more diagnostic value than assays that cannot distinguish whether a fly would not walk or cannot even walk. Modern assays have developed a suite of performance parameters that facilitate such discrimination (e.g., Ctrax, CADABRA; Simon and Dickinson, 2010) by using basic locomotory parameters (position, velocity, acceleration) to compute higher-order behavior (e.g., distance between flies, angle between flies' orientation, see BehavioralMicroarray MATLAB Toolbox of Ctrax).

\section{This Study}


We aimed to develop an assay that combines the strengths of a simple reactivity-based assay (the tap-down) with the strength of an activity-based assay (walking arena) by adding a mechanical challenge to the arena (sloped walls). The goal was to develop an assay (1) that could differentiate between changes in motor ability and internal motivation, (2) that was amenable to automated tracking, and (3) that has high statistical power (can achieve significant results with a low number of flies).

\section{BMAA-A Case Study on a Neurotoxin and Glutamate Agonist}

In order to test our new behavioral assay, we focused on quantifying the effects of the environmental neurotoxin $\mathrm{B}-\mathrm{N}$-methyl amino L-alanine (BMAA). BMAA is a non-natural amino acid that has been implicated in the neurodegenerative disease called amyotrophic lateral sclerosis-Parkinsonism dementia complex (ALS-PDC) in humans (Whiting, '88; Cox et al., 2003). However, the exact mechanism by which BMAA induces the ALS-PDC associated locomotory defects is not understood. Cellular studies in cultured mammalian cells indicate that BMAA acts as a glutamate agonist: the effects of BMAA are attenuated by competing chemicals that bind to the glutamate-associated NMDA and AMPA receptors (Weiss et al., '89; Rao et al., 2006). Animal model studies (Karamyan and Speth, 2008) using mice, rats (Karlsson et al., 2011), cynomolgus monkeys, and fruit flies (Zhou et al., 2009; Zhou et al., 2010) all show an apparent locomotor phenotype. While qualitative observations are sufficient to summarize all of the gross defects in locomotion into a defined phenotype, we needed quantitative measures to discern dose effects and the progression of defects.

In the case of BMAA, we also needed performance measures that can tease apart central and peripheral nervous system effects. Glutamate is the main excitatory neurotransmitter at the neuromuscular junctions of insects (Usherwood, '94). It is also a neuromodulator in the central complex (Featherstone et al., 2005), a structure that controls walking activity levels and walking speed (Strauss and Heisenberg, '93; Martin et al., '99b). If BMAA is a glutamate agonist, then it should affect both motor ability and internal motivation. First, due to its excitatory effect at the neuromuscular junctions, BMAA should cause a loss of fine motor control and hence should lower walking speed or even prevent walking entirely. Second, due to its excitatory effect on the locomotory control centers in the central nervous system, BMAA should cause an increase in walking activity and walking speed.

\section{METHODS}

\section{Insects and Neurotoxin Treatment}

We used laboratory strains of D. melanogaster, wild-type Canton S. We selected only virgin females to avoid aggressive and courtship displays, mating and maternal behavior. Breeding stock flies were raised on food composed of cornmeal, sucrose, yeast, agar, propionic acid, nipagen, and green food coloring. For the experiments, we transferred virgin female flies 1 to 2 days after eclosion into four treatment vials at 10 flies per vial. The treatment vials contained food composed of agar, yeast extract, sucrose, glucose, magnesium sulfate, calcium chloride, 
and propionic acid, plus $0,12.5,25$, or 50 mM BMAA. The flies in the treatment vials were kept on a $12: 12 \mathrm{hr}$ light cycle at $22^{\circ} \mathrm{C}$.

We recorded the flies' behavior at 2:00 PM (from 6 to $6.5 \mathrm{hr}$ into the light cycle) 1, 2, and 3 days after the switch to food containing BMAA. We used two different behavioral assays, the tapdown and the lenticular arena. For the experiments in the lenticular arenas, the flies were transferred to the arenas in batches of five flies per arena. For the tap-down assay, flies were transferred in batches of 10 to the tap-down vials. To transfer the flies, they were cold anesthetized for $2 \mathrm{~min}$ in a $-20^{\circ} \mathrm{C}$ freezer. Experiments commenced $15 \mathrm{~min}$ after transfers, allowing reactive behaviors in response to the transfer to subside. After the recordings, the flies were again cold anesthetized and transferred back into the food vials.

\section{Filming Set-ups and Procedure}

Tap-Down. The tap-down setup consisted of four cylindrical vials $(84 \mathrm{~mm}$ high, $20 \mathrm{~mm}$ diameter) glued in a row onto a strip of Plexiglass. This arrangement enabled us to tap down four vials simultaneously and to position them reproducibly for video recording from a side view. Each vial was closed with a plastic stopper, leaving an internal height of $80 \mathrm{~mm}$. To record the tap-downs, we used a Photron PCl camera (1024×1024 pixels; Fastcam $1024 \mathrm{PCl}$, Photron USA, San Diego, CA), recording at 10 frames per second with a 50-mm lens (Zeiss Planar T 1.4; F stop 5.6; Carl Zeiss AG, Jena, Germany). A function generator (Grass S44 Stimulator; Grass Technologies, West Warwick, RI) was used to trigger the cameras at this low rate. Illumination was provided by 2 halogen lamps ( $250 \mathrm{~W}$ ), with a Plexiglas diffuser. The flies were tapped down with four quick taps, and their climbing success was scored $10 \mathrm{sec}$ after the last tap. We recorded a total of six experimental runs using a total of 240 flies, 60 flies per treatment (10 flies per vial).

Lenticular Arena. The lenticular arena was circular $(75 \mathrm{~mm}$ in diameter and $8 \mathrm{~mm}$ high at its deepest point). Its floor was made from a watch glass ( $75 \mathrm{~mm}$ diameter; Catalog number 02610 C, Fisher Scientific, Pittsburgh, PA) glued inside a PVC plumbing adapter (NIBCO 3" x 1-1/2" reducer bushing, model number 5801-2-F; NIBCO, Inc., Elkhart, IN). The lenticular floor also created an arena with no side walls (Fig. 1). In our particular design, we did not use any chemical boundaries to prevent flies from climbing onto the ceiling (unlike Simon and Dickinson, 2010). While the lenticular shape avoided any visual occlusion issues due to walls, there remained the issue of flies overlapping visually when the path of a fly on the floor crossed the path of a fly on the ceiling. Such overlaps could cause the automated tracking software to mistrack, so we used a low number of flies per arena to lower the probability of such events.

Each arena was painted matte black, and covered with a flat, circular antireflection-coated glass window (Edmund Optics, Barrington, NJ). To record all four treatment groups simultaneously, four arenas were placed under one camera. The arenas were covered with a hemispherical plastic diffuser illuminated by an outer ring of eight red LEDs (Luxeon Star with collimating optics, $1 \mathrm{~W}$, wavelength $660 \mathrm{~nm}$; Philips Lumileds Lighting Company, San Jose, CA). As shown in 
Figure 1, the camera viewed the arenas through an opening at the top of the diffuser. Additional black baffles (not shown) were used to reduce lens flare. The resulting images were free of interfering reflections, highlights, or shadows.

All video recordings were made with a Photron PCl camera $(1024 \times 1024$ pixels $)$ and a $50-\mathrm{mm}$ lens (Carl Zeiss, Planar T 1.4 operating at $f / 5.6$ ). The image of a single fly under these conditions was typically 25 pixels from anterior head to posterior abdomen. Flies were recorded for $10 \mathrm{~min}$ at a frame rate of $10 \mathrm{~Hz}$ (exposure time $125 \mathrm{~ms}$ ). We recorded a total of 40 flies: 10 flies per treatment and 5 flies per arena.

\section{Data Analysis}

Tap-Down. To quantify the flies' climbing performance, we counted the number of flies that climbed past the $60 \mathrm{~mm}$ mark within $10 \mathrm{sec}$. The time count started as soon as the tapping down was complete and the vials were sitting flat on the lab bench. Climbing success was expressed as fraction of the 10) flies in a vial that passed the predefined mark.

Lenticular Arena. All recordings from the lenticular arenas were processed using Ctrax (Branson et al., 2009) to track the $x$, y position of flies in the arena automatically. Ctrax cannot differentiate between flies walking on the ceiling vs. the floor, so this parameter was scored manually. We are in the process of automating this procedure, exploiting the fact that the ventral side of flies is lighter than their dorsal side (code available on request from UKM). Using custom programs written in MATLAB (v. 2008a, with image processing toolbox, MathWorks, Natick, MA), we computed radial distance from the arena center, walking speed, and the activity levels of each fly over time (MATLAB scripts available on request from UKM).

Radial distance served as an indicator for climbing performance. With the slope of the circular arena floor gradually increasing from $0^{\circ}$ at the center to $15^{\circ}$ at the edge, the proportion of total time spent at a particular radial distance can serve as a measure of climbing ability. We obtained radial distance by converting the Cartesian output of Ctrax into radial coordinates with the center of the arena as the origin. For statistical analysis, the per-frame radial distances of each fly were tabulated into annular bins of equal area. The resulting histogram represents the sample radial density function of fly positions over the entire 10-min observation period. The slope of the arena was shallow enough that foreshortening was negligible (cos (slope angle) $>0.96$ ). Therefore the floor of the arena was assumed to be flat when transforming coordinates and calculating bin surface areas. In order to calculate per-fly average radial distance, each bin was represented by its average radial distance from the center of the arena. If the flies were randomly distributed within the arena, then the amount of time spent in each bin would be identical, resulting in a flat radial density function of $4.8 \%$ per bin (for 21 floor bins). Spending more than $4.8 \%$ of the time in a particular bin indicated a preference, spending less than $4.8 \%$ indicated that the fly avoided this particular radial bin. Flies on the ceiling were tabulated in a separate outer bin, again normalized for surface area, with a radial distance arbitrarily assigned as equivalent to the edge of the arena. 
We conducted the statistical analysis for 21 and 11 floor bins to confirm that our results did not depend on the number of bins (MATLAB algorithms available from UKM). This analysis showed that halving the number of floor bins did not affect the average radial position value, the difference among groups or the $\mathrm{P}$ value. The choice of number of bins was based on Valente et al. (Valente et al., 2007); we maintained a similar ratio of data points to number of bins in our study ( $10^{3}$ data points per bin).

To compute activity level (the percentage of time spent active) and walking speed, a fly was defined as active if its speed was greater than $2 \mathrm{~mm} \mathrm{~s}^{-1}$ for more than 5 frames ( $=0.5 \mathrm{sec}$ ); this threshold was defined to exclude small movements, for example during grooming, from being counted as walking.

Statistical Analysis. Most of our data were not normally distributed, they could not be transformed to a normal distribution, or they violated some of the assumptions of ANOVA. If the data from at least one of the three recordings in a data set were not normally distributed, we then used the most powerful statistical test whose assumptions were met by our data set. If the data were independent, normal, and met homogeneity of variance assumptions (tapdown), then we used ANOVA with a Tukey's post hoc test for among group comparisons. If the data violated the assumption of homogeneity of variance (radial distance, walking speed), yet the data remained normal and independent, we used ANOVA with a Games-Howell post hoc test, which does not assume equal variance for among group differences. If the data were independent but any of the groups being compared were not normal (activity level), a nonparametric Kruskal-Wallis ANOVA test was applied with Mann-Whitney $U$ test for pairwise comparison among groups. In all cases for multiple comparisons, we used a Bonferroni correction to adjust the $\mathrm{P}$ value appropriately. Data processing and statistical analyses were carried out using custom software in MATLAB and SPSS (SPSS, Inc., Chicago, IL). Flies died from the BMAA treatment or were lost during the experimental procedure, so that the $n$ values in the results section were not identical for all groups on all days.

\section{RESULTS}

\section{Comparison of Tap-Down vs. Lenticular Assay-Assessment of Climbing Ability}

Flies did not distribute randomly: the flies climbed to higher positions both during reactive locomotion in response to a tap-down and during active (spontaneous) locomotion in the lenticular arena. In the tap-down assay, the flies raced to the top of the vial after being tapped down (Fig. 2)-86\% of the control flies were in the top part of the vials $10 \mathrm{sec}$ later (Table 1). In the lenticular arena, the flies favored the ceiling and the high edge of the lenticular floor (Figs. 3 - 4, Table 2): the control flies spent more than $50 \%$ of the recorded $10 \mathrm{~min}$ on the ceiling or at the edge of the arena-defined as the outer $3 \mathrm{~mm}$ of the $37 \mathrm{~mm}$ radius arena, which put the flies within 1 body length of the edge. So climbing behavior was visible in the tap-down, a reactivity-based assay with a strong stimulus (tapping down), as well as in the lenticular arena, an activity-based assay with a weak stimulus (slope). The lenticular arena also found 
considerable thigmotaxis - the flies favored the edges of both the arena floor and ceiling (Fig. 2).

Both assays showed statistically significant treatment effects ( = difference among control and treated flies) (Tables 1 - 2). The differences between controls and BMAA-treated flies were still weak after 1 day of treatment-only the flies at the highest dose climbed significantly less well, but the medium dose $(25 \mathrm{mM})$ flies were not significantly different from the control flies. The tap-down with its vertical slope found statistically significant treatment effects for all three doses already on Day 2 (Table 1). In the lenticular arena with its more gentle slope, the flies treated with the lowest dose $(12.5 \mathrm{mM})$ remained statistically identical to the controls even on Day 3 (Table 2). So the tap-down has a lower detection limit than the lenticular arena for treatment effects, it detected even a slight loss of motor ability.

The two assays differed also in their ability to detect dose effects ( = difference among groups treated with different doses). A higher dose should cause a more severe loss of motor ability. Two treated groups that differ less in their dose should be less likely to be statistically different that two treated groups whose doses differ a lot. The lenticular arena follows this prediction consistently. The lenticular arena found, first, that flies treated with the lowest dose were not statistically different from the controls; second, that doubling the dose ( 12.5 to $25 \mathrm{mM}, 25$ to $50 \mathrm{mM}$ ) also did not produce a significant change. However, the lenticular arena always found a significant difference between groups that were separated by one dose (e.g., 0 vs. $25 \mathrm{mM}, 12.5$ $\mathrm{mM}$ vs. $50 \mathrm{mM}$ ). Summed up over all three days of experiments, the lenticular arena found no significant differences between "adjacent" treatment groups (e.g., 12.5 vs. $25 \mathrm{mM}$ ) in nine out of nine pair-wise comparisons. Yet it found a significant difference between "separated" groups in six out of six pair-wise comparisons (e.g., 12.5 vs. 50 mM). In contrast, the tap-down found that "adjacent" treatment groups were statistically identical in only five out of nine possible cases, while groups separated by one dosage group were statistically different in only four out of six possible cases. The tap-down mainly identified that controls were statistically different from treated flies (treatment effect), but it was less able to differentiate between different doses than the lenticular arena.

The lenticular arena was also stronger at identifying a possible progression effect. From Day 1 to Day 3, the lowest dose stopped being statistically different from the medium dose, and the medium dose stopped being statistically identical to the controls. The tap-down showed only weak indications of a progression effect (Table 1). On Day 1, only the flies feeding on the highest dose were significantly different from the control. By Day 2, all three toxin-treated groups were significantly different from the control.

To sum up, the tap-down and arena showed a trade-off between a low detection limit to identify treatment effects and high sensitivity for dose and progression effects. An assay that presented a severe physical challenge (vertical slope) to ensure a low detection limit for treatment effects (even a low dose will cause a significant decline in performance) is less able to detect dose effects (low-dose flies perform similarly poorly to high-dose flies) or progression 
effects. The lenticular arena with its gentle slopes was better able than the tap-down to capture the gradual loss of motor ability caused by a gradual increase in dose or prolonged exposure.

The main difference between the assays was their statistical power. Despite the arena using far fewer flies than the tap-down, it achieved similar statistical results as the tap-down. The tapdown used 240 flies, the lenticular arena used only 40 flies. The main reason for the large difference in statistical power was the experimental design. In the tap-down, the experimental unit was the vial, which contained 10 flies. In the lenticular arena, the experimental unit was the fly. This difference caused the tap-down to have a much lower sample size despite the larger number of flies.

While the tap-down found no heterogeneity in the data, the lenticular arena showed that there was considerable variation within each treatment group. Tests for homogeneity of variance indicated non-equal variance: there were two types of flies present within a given treatment group, climbers and non-climbers (Figs. 3 - 4). Some flies spent most of their time near or on the ceiling, while other flies spent considerable time at lower levels-they struggled to maintain their position near or on the ceiling and regularly fell off or rolled down the slope. Some of the density functions of radial position were bimodal (Fig. 4, flies treated with $50 \mathrm{mM}$, Day 1 of treatment), giving a visual impression of this heterogeneity. Examination of the recordings confirmed that two subpopulations of flies were present, rather than all flies sharing a bimodal preference for position. Some flies maintained near normal motor ability and were able to climb to areas near the edge of the arena; other flies were more strongly affected by the toxin and remained near the lowest point at the center of the arena.

\section{Lenticular Assay-Assessment of Walking Speed and Activity Levels}

We developed the lenticular assay as an alternative to the tap-down with the goal of quantifying not just climbing ability, but also other performance parameters. Given that the central complex of fruit flies controls internal locomotor motivation both through affecting activity level and walking speed (Strauss and Heisenberg, '93), we were particularly interested in parameters that would allow us to differentiate between changes in motor ability ( = effects on peripheral locomotory system) and motivation ( = effects on the central complex). So we assessed climbing performance, activity levels, and walking speed. Climbing performance was quantified as radial distance and it is predominantly a measure of motor ability. Activity levels were quantified as percentage of time spent walking at a minimum speed of $2 \mathrm{~mm} \mathrm{~s}-1$ and this performance parameter is predominantly a measure of motor motivation. Walking speed is a performance parameter that is affected strongly by both motor ability and motivation (Strauss, 2002).

To assess internal motivation, we quantified activity levels (= percentage of time spent walking). The raw walking path data (Fig. 3) already hinted at differences in activity levels between the four groups of flies. Our statistical analysis of activity levels showed that treated flies differed significantly from the control group (Table 3, Fig. 5). While the control flies were 
active for $37 \pm 13 \%$ of the time (mean $\pm S D ; n=10$ ) at 1 day after treatment, the treated flies were active for $64 \pm 27$ to $87 \pm 5 \%$ of the time $(n=10)$. So, 1 day of treatment caused significant hyperactivity in the treated flies at all three doses. Compared with Day 1 of treatment, the control flies became more active during Days 2 and 3, while the BMAA-treated flies either maintained their elevated activity levels (lowest dose) or became less active (medium and high dose). This progression effect mirrored the dose effect. Higher dose and longer exposure both suppressed the initial hyperactivity. We hypothesize that this suppression was caused by decreasing motor ability. This loss of motor ability manifested in the flies' use of space: flies spent more time near the center of the arena and were unable to climb onto the ceiling of the dish (Table 2, Figs. 3 - 4).

We also quantified walking speed, a performance parameter that depends both on motor ability and motivation, but that is more sensitive than activity level to changes in motor ability. We found that walking speed was significantly elevated compared with the control $(5.7 \pm 0.9, \mathrm{n}$ $=10$ ) only at the lowest dose and only on Day $1(6.4 \pm 1.0, n=10)$ (Table 4, Figure 5). All other doses on all days showed either a significant decrease in walking speed or no significant difference (medium dose on Day 1 ) from the controls. We hypothesize that this decline in walking speed was again caused by decreasing motor ability.

\section{DISCUSSION}

Behavioral assays are designed to help find differences between controls and treated groups by (1) eliciting behaviors that facilitate discrimination and (2) by recording parameters that can quantify those behaviors. The lenticular arena adds climbing to the range of behaviors sampled by the commonly used flat arenas. The lenticular shape also allows radial position to become a straight-forward measure of climbing performance. So a lenticular floor can be a useful addition to a circular arena assay that screens motor behavior to assess locomotor competence.

\section{Experimental Design for Behavioral Arenas}

Arenas are powerful behavioral assays because they enable experimenters to record behavior for extended periods of time. Arenas are more powerful than the tap-down assay for several reasons. Recording behavior for an extended period of time gives arenas (1) higher statistical power and (2) a wider range of quantifiable performance parameters. This range is not only useful to (2a) characterize behavioral phenotypes. It is also useful for (2b) peeking inside the black box that is the fruit flies' motor control.

Statistical Power. Recording behavior at multiple time points enables experimental designs in which the fly, rather than the arena, is the experimental unit. One tap-down experiment yields a sample size of one, despite each vial containing typically 10 to 40 flies (e.g., Feany and Bender, 2000; Ling et al., 2009). Tapping down the same flies repeatedly does not increase sample size. Instead, studies need to rely on multiple experiments, usually requiring more than 100 flies per treatment group (Feany and Bender, 2000; lijima et al., '04; Ling et al., 2009). Compared with the tap-down, the arena was more efficient-it reached a sample size larger 
than 1 without requiring many replicate experiments. In our study, we used 240 flies (60 per treatment group) in the tap-down, compared with 40 flies (10 per treatment group) in the arena. Despite the large difference in the number of flies, arena, and tap-down detected similar effect sizes at similar levels of significance. On the downside, the arena required nonparametric tests and the data were less precise due to a larger variation.

Several refinements of the tap-down have been developed (e.g., Benzer, '67; Greene et al., 2003) to improve the sensitivity of this assay, such as the stress-resistance assay (Ruan and Wu, 2008). This assay is similar to the lenticular arena in that it was designed to study and encourage locomotory behavior over a prolonged time period rather than at one time point. These more complex tap-down procedures, which sample performance at multiple points in time, should be able to reach a similar statistical power to an arena.

Behavioral assays also should quantify parameters that have a high signal-to-noise ratio and are therefore more likely to identify statistically significant differences. Radial distance is such a low-noise parameter because it integrates position information. However, the lenticular arena itself did not generate as strong a signal as the tap-down due to its gentler slope presenting a less severe physical challenge. This lower effect size of the lenticular arena gave the tap-down a lower detection limit (treatment effect) - at the expense of lower sensitivity (dose effect). The detection threshold of both assays could be adjusted by adjusting the slope-a lenticular arena with steeper slopes should have a lower detection limit. The lenticular assay could also adjust its sensitivity by adjusting the gradient of the change in slope-a more dramatic increase in slope from the center to the edge of the arena should decrease sensitivity. The tap-down can adjust its detection threshold, but increasing its sensitivity requires a more drastic redesign (e.g., staggered tap-down; Benzer, '67).

Experimental designs, such as arenas, in which the fly is the experimental unit, are desirable particularly when treatments are difficult to administer to a large number of flies (injection of the toxin), or when sample size is limited by poor fecundity or survival. Radial distance from the center of a lenticular arena is a performance parameter that has a high signal-to-noise ratio, is readily obtained by automated tracking, and can be adjusted by a steeper increase in slope to make the assay more sensitive for treatment effects (detecting significant differences between control and treated flies), yet less sensitive for dose effects (detecting significant differences between different doses).

Diagnostic Power through Gathering Multiple Parameters. Behavioral assays must be able to elicit behaviors that can be quantified in ways that facilitate discrimination. Reactivity-based assays (such as the tap-down) can be powerful yet simple screening tools because their selection of behavior (climbing), environment (vertical wall), and performance parameter (ability to reach a predefined height) all facilitate discrimination. Reactivity-based assays reduce intra-individual variation and heterogeneity, simplifying statistical analysis. If the reactive behavior takes place in an environment that presents a strong physical challenge, the assay will have a low detection limit for locomotory deficits. All these aspects make the tap-down well 
suited for detecting treatment effects in toxicological screens. However, by focusing on a single, reactive behavior, the tap-down is less able to detect dose effects, neither can it provide a comprehensive picture of a locomotor phenotype. These limitations make the tap-down less suitable for pharmacological and mutational analysis. Therefore, screening analyses interested in multifactorial effects often combine a tap-down with other, activity-based assays (e.g., Ruan and $\left.\mathrm{Wu},{ }^{\prime} 08\right)$ to quantify additional locomotory parameters.

Activity-based assays, such as lenticular or flat-bottomed arenas (Valente et al., 2007; Simon and Dickinson, 2010), allow flies to display a wider range of behaviors, including spontaneous behavior, enabling the experimenter to quantify multiple parameters in order to build a locomotor phenotype with a single multi-parameter assay. Arenas are usually designed to record behavior over an extended period, which facilitates the collection of a range of behavioral characteristics, such as activity levels (Table 3, Fig. 5), space use (Fig. 4) and walking speed (Table 4, Fig. 5). Through the post-processing routines available e.g., for Ctrax and CADABER, further locomotory characteristics can be determined, such as walking direction, path length, and path curvature (Branson et al., 2009; Dankert et al., 2009; Simon and Dickinson, '10), or other spatial preferences (Valente et al., 2007). Arena designs are pushing towards facilitating automated data recording and analysis as well as producing behaviors that facilitate quantification of multiple performance indicators (e.g., Albrecht and Bargman, 2011, Pandey and Nichols, 2011). If activity-based arenas want to assess motor ability unambiguously, they must present a physical challenge.

Diagnostic Power through Combining Parameters. A flat-bottomed arena (e.g., Simon and Dickinson, 2010) facilitates automated data recording and analysis. Yet, without providing a physical challenge, it is less sensitive to changes in motor ability. The tap-down, like all reactivity-based assays, provides a strong external stimulus that confounds quantification of internal locomotor motivation. A lenticular arena combines aspects of the tap-down with aspects of the flat-bottom arena assay. Its lenticular floor provides a physical challenge that allows quantification of walking ability. Yet the physical challenge is also weak enough to allow internal locomotory motivation to be quantified.

This ability to discriminate between effects on locomotory ability and internal motivation was exemplified by our case study on BMAA. We predicted that BMAA should affect both the central and peripheral nervous system, causing motor ability to deteriorate while increasing motivation. We chose to quantify activity levels, walking speed, and climbing performance as our three performance parameters to assess ability and motivation. Both tap-down and lenticular arenas were able to capture the loss of motor ability by quantifying the climbing ability. However, the tap-down assesses a reactive type of climbing behavior and therefore could not quantify effects on internal motivation (activity levels). The lenticular arena allowed us to quantify activity levels and walking speed. Both parameters indicated that BMAA increased motivation (the flies walked faster and more often). However, a flat-bottomed arena would not be able to quantify climbing ability as a measure for motor ability. Instead it would 
have to rely solely on walking speed, an indicator of walking ability that is confounded by motivation. If speed were used as purely an indicator for ability, our data would suggest that BMAA in low doses actually improved walking ability-flies treated with the lowest dose walked significantly faster than control flies on Day 1 . The combination of climbing success (radial distance), walking speed, and activity data showed that BMAA probably affected both motivation and ability. Flies treated with the lowest dose maintained elevated activity levels even on Day 3. But even at the lowest dose, climbing ability began to decline. Walking speed showed the combined effect of increased motivation battling against declined ability-after an initial increase on Day 1, walking speed declined steadily on Day 2 and 3 in the lowest dose flies. We hypothesize that at higher doses and after prolonged exposure, the excitatory effects of BMAA on the central pattern generator are obscured by BMAA causing a loss of motor ability.

Arenas are becoming an important diagnostic tool not just in screening, but in all areas of behavioral research as techniques are developing to manipulate flies remotely rather than tethering them to electrodes (e.g., Lima and Miesenböck, 2005), and as technologies become available that can track freely moving flies (e.g., Fry et al., 2003; Branson et al., 2009; Ristroph et al., 2009; Simon and Dickinson, 2010). The lenticular arena is a simple variation on an arena assay. By including a physical challenge in the form of a gradually increasing slope, it can measure an additional performance parameter (walking ability) compared with a flat-bottomed arena. By characterizing this performance parameter by the fly's radial distance from the arena center, the arena makes this parameter easy to quantify. Furthermore, its sensitivity is easily adjusted through the concavity of the floor to the strength of the expected effect or the goal of the study.

\section{GRANT STATEMENT}

Grant Sponsor: NSF-MRI; Grant Number: DBI-0821820.

N.T.M. and J.J.G. contributed equally to this manuscript.

\section{ACKNOWLEDGMENTS}

The following students and volunteers contributed to this research: Ana Soltani, Abeer Qureshi, Jennifer Lei, Farzad Mazloomi, Tina Sakha, Michael Adam, Chihiro Saito, Shereen Dimes, Simi Sharma, Athena Goodarzi.

\section{CONFLICT OF INTEREST}

The authors report no conflict of interest. 

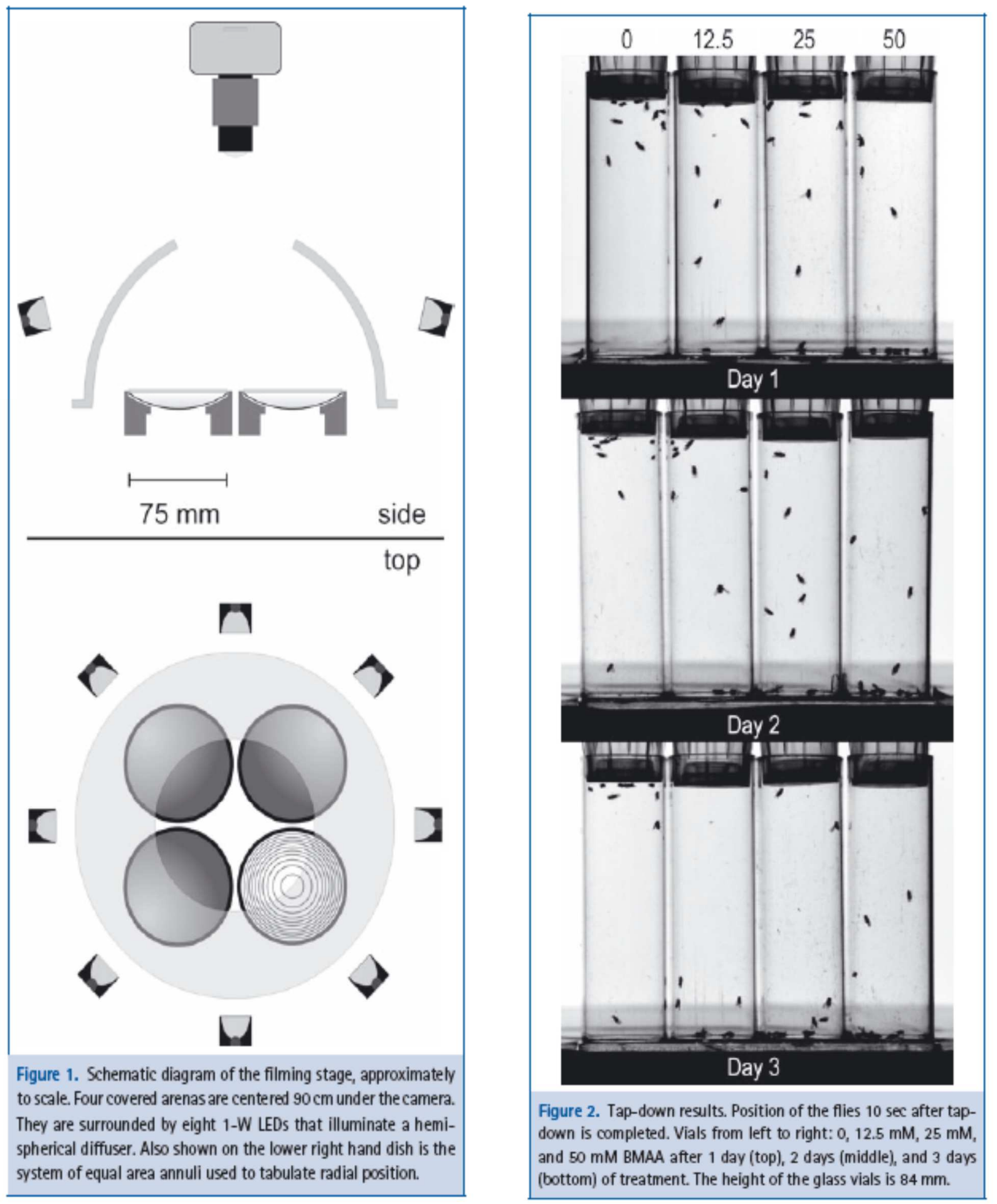


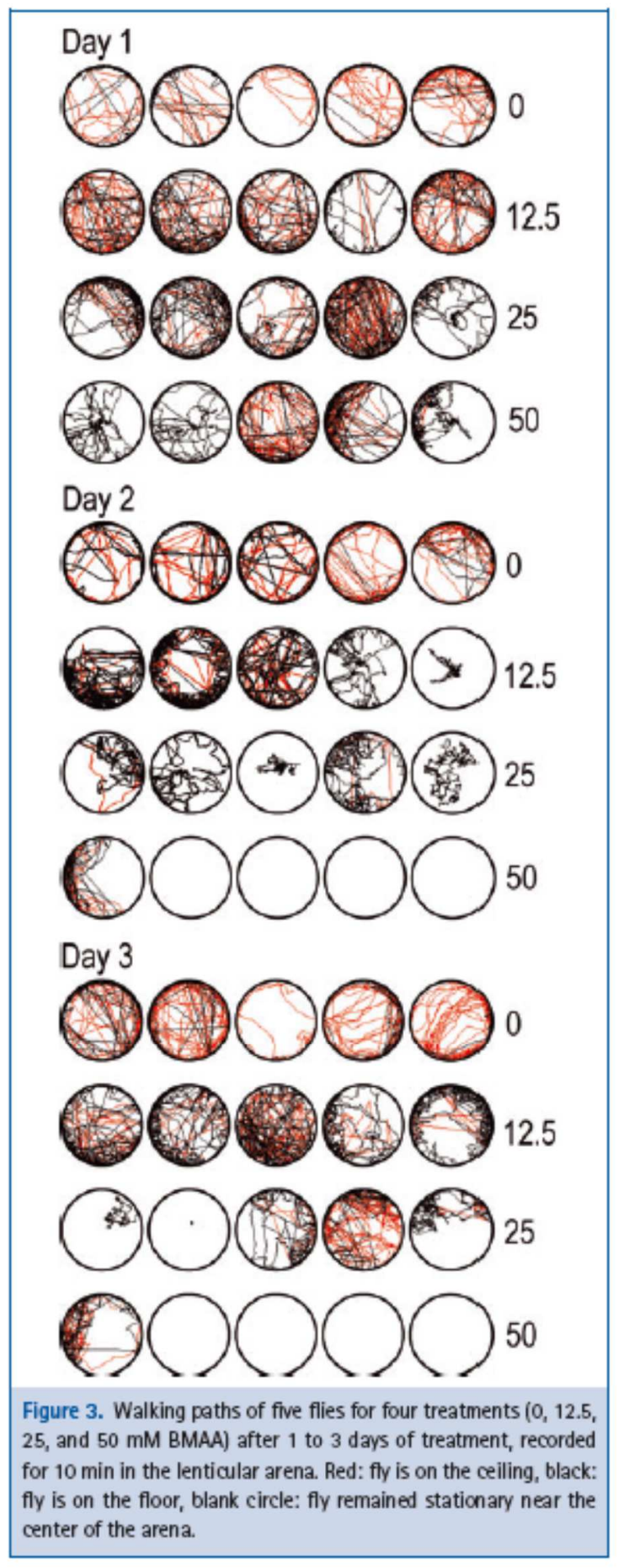

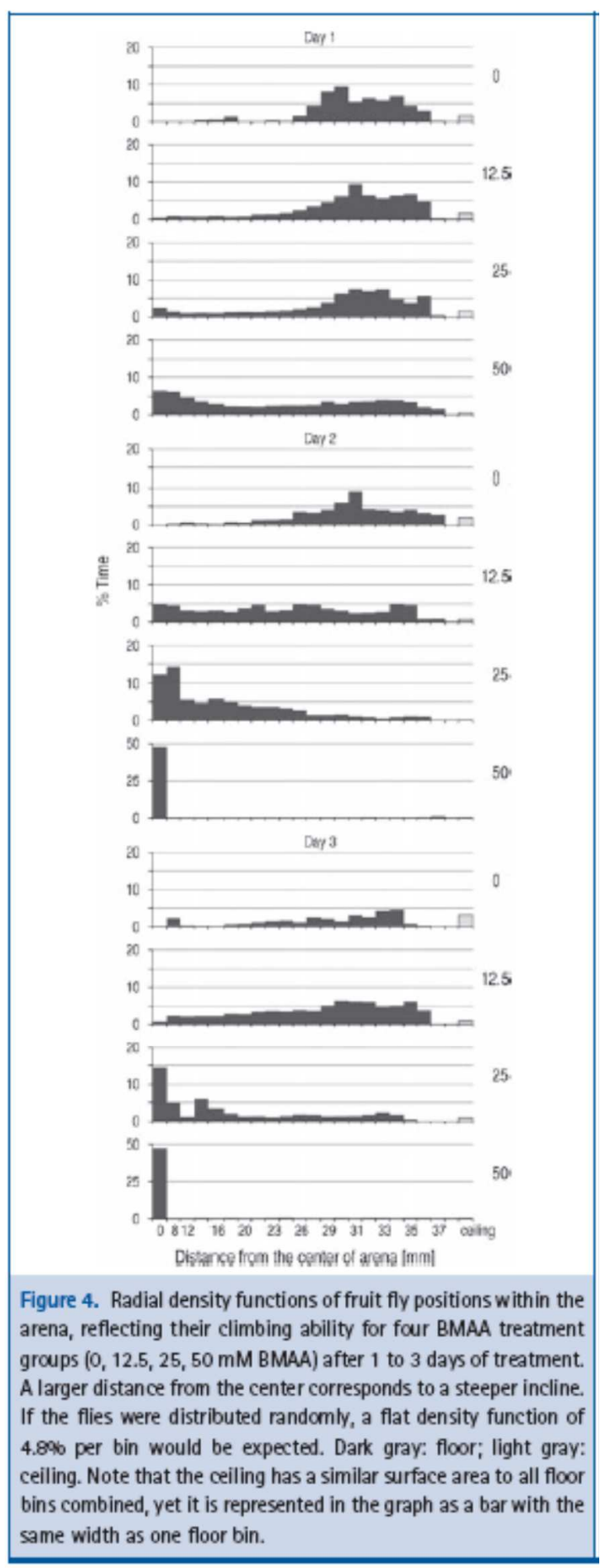




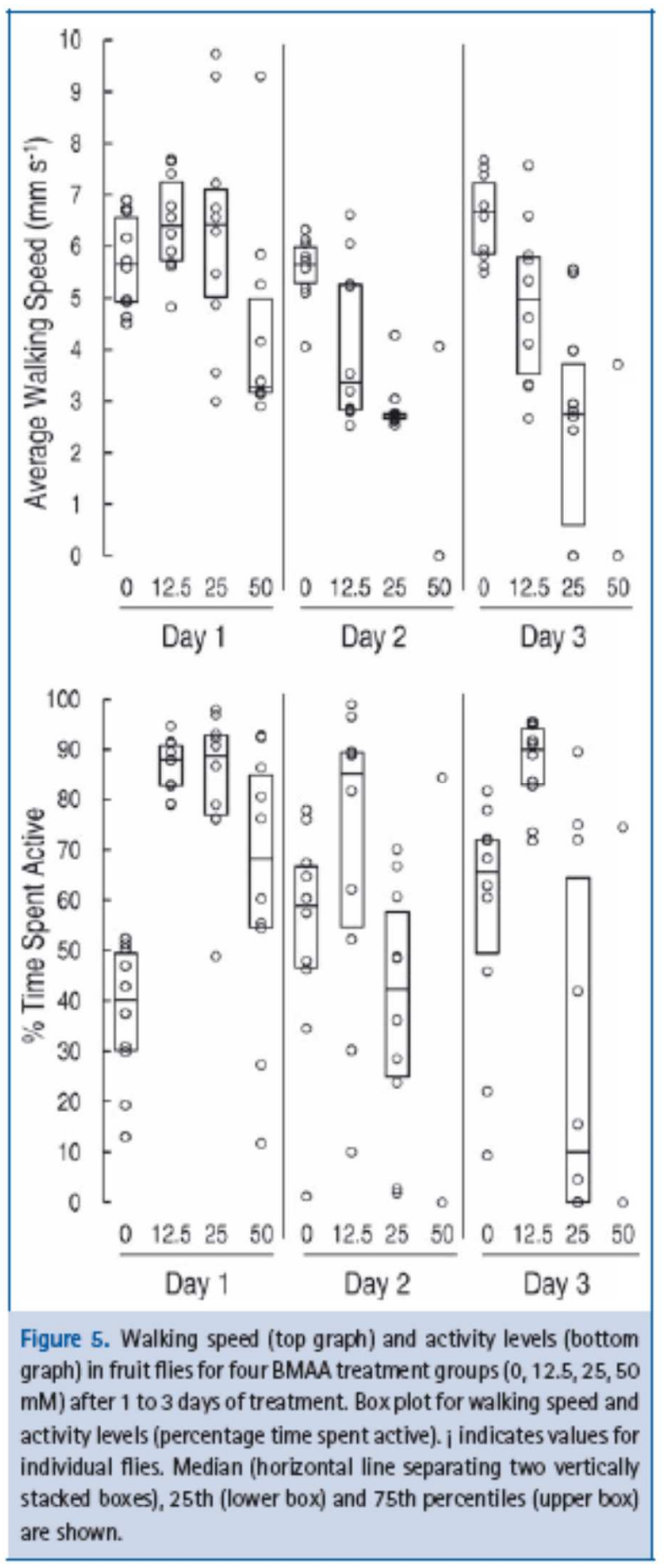




\begin{tabular}{|c|c|c|c|}
\hline \multirow{2}{*}{$\begin{array}{l}\text { Treatment } \\
(\mathrm{mM})\end{array}$} & \multicolumn{3}{|c|}{ Duration of treatment (days) } \\
\hline & 1 & 2 & 3 \\
\hline & \multicolumn{3}{|c|}{ Climbing performance $(\$ b)$} \\
\hline 0 & $85.6 \pm 7.0(5)^{2}$ & $85.5 \pm 12.3(5)^{2}$ & $85.9 \pm 7.5(6)^{2}$ \\
\hline 12.5 & $78.9 \pm 7.8(5)^{2}$ & $57.5 \pm 16.0(5)^{b}$ & $38.9 \pm 16.3(6)^{p}$ \\
\hline 25 & $66.5 \pm 13.1(5)^{2}$ & $38.9 \pm 4.5(5)^{p}$ & $25.0 \pm 15.8(6)^{p}$ \\
\hline 50 & $14.8 \pm 8.9(5)^{b}$ & $15.6 \pm 8.0(5)^{2}$ & $5.1 \pm 4.4(6)^{b}$ \\
\hline \multicolumn{4}{|c|}{$\begin{array}{l}\text { Cimbing performance was calculated by the percentage of flies that } \\
\text { climbed } 60 \mathrm{~mm} \text { within a } 10 \text {-sec period after tap-down. All data are pre- } \\
\text { sented as mean } \pm S D \text { ( } n=\text { number of vials per treatment). The results of } \\
\text { statistical tests are indicated by letter codes, } P<0.05 \text {; treatment groups } \\
\text { with the same letter codes are not statistically different. }\end{array}$} \\
\hline
\end{tabular}

\begin{tabular}{|c|c|c|c|}
\hline \multirow[b]{2}{*}{ Treatment $(\mathrm{mM})$} & \multicolumn{3}{|c|}{ Duration of treatment (days) } \\
\hline & 1 & 2 & 3 \\
\hline & \multicolumn{3}{|c|}{ Radial distance (mm) } \\
\hline 0 & $30.88 \pm 2.03(10)^{2}$ & $29.50 \pm 2.52(10)^{2}$ & $29.30 \pm 3.16(10)^{2}$ \\
\hline 12.5 & $30.66 \pm 1.92(10)^{2}$ & $22.92 \pm 7.60(10)^{2}$ & $27.27 \pm 4.30(10)^{2 t}$ \\
\hline 25 & $27.13 \pm 7.01(10)^{2 h}$ & $14.76 \pm 6.52(10)^{2 h}$ & $18.87 \pm 6.54(10)^{\mathrm{bc}}$ \\
\hline 50 & $22.83 \pm 5.96(10)^{\mathrm{b}}$ & $10.57 \pm 9.19(10)^{b c}$ & $10.44 \pm 6.90(10)^{\mathrm{c}}$ \\
\hline \multicolumn{4}{|c|}{$\begin{array}{l}\text { Radial distance was calculated as the distance between the fly and the center of the arena, assuming that a position on the ceiling equals the highest } \\
\text { position (= outer radius of the arena) achievable on the floor. The radius of the arena is } 37.5 \mathrm{~mm} \text {. All data were presented as mean } \pm \text { SD ( } n=\text { number of } \\
\text { flies per treatment). The results of statistical tests are indicated by letter codes, } P<0.05 \text {; treatment groups with the same letter codes are not statistically } \\
\text { different. }\end{array}$} \\
\hline
\end{tabular}

\begin{tabular}{|c|c|c|c|}
\hline \multirow[b]{2}{*}{ Treatment (mM) } & \multicolumn{3}{|c|}{ Duration of treatment (days) } \\
\hline & 1 & 2 & 3 \\
\hline & \multicolumn{3}{|c|}{ Total time spent active $(\$ 6)$} \\
\hline 0 & $37.4 \pm 13.8(10)^{2}$ & $53.3 \pm 22.7(10)^{2}$ & $57.2 \pm 24.2(10)^{2 \mathrm{~b}}$ \\
\hline 12.5 & $86.7 \pm 5.4(10)^{b}$ & $70.0 \pm 30.5(10)^{2}$ & $869 \pm 8.8(10)^{b}$ \\
\hline 25 & $83.8 \pm 14.7(10)^{\mathrm{pc}}$ & $38.8 \pm 24.6(10)^{\mathrm{ab}}$ & $29.9 \pm 36.4(10)^{p c}$ \\
\hline 50 & $63.8 \pm 27.5(10)^{c}$ & $8.5 \pm 26.7(10)^{p}$ & $7.5 \pm 23.5(10)^{c}$ \\
\hline
\end{tabular}

\begin{tabular}{|c|c|c|c|}
\hline \multirow[b]{2}{*}{ Treatment (mM) } & \multicolumn{3}{|c|}{ Duration of treatment (days) } \\
\hline & 1 & 2 & 3 \\
\hline & \multicolumn{3}{|c|}{ Average walking speed $\left(\mathrm{mm} \mathrm{s}^{-1}\right)$} \\
\hline 0 & $5.68 \pm 0.91(10)^{\mathrm{abc}}$ & $5.55 \pm 0.65(10)^{2 b}$ & $6.56 \pm 0.81(10)^{\mathrm{bb}}$ \\
\hline 12.5 & $6.44 \pm 0.96(10)^{p}$ & $4.09 \pm 1.54(10)^{p}$ & $4.91 \pm 1.58(10 p$ \\
\hline 25 & $6.28 \pm 2.19(10)^{\mathrm{abc}}$ & $2.89 \pm 0.51(10)^{p c}$ & $2.59 \pm 2.09(10)^{t b}$ \\
\hline 50 & $4.35 \pm 2.01(10)^{r}$ & $0.41 \pm 1.29(10)^{c}$ & $0.37 \pm 1.18(10)^{r}$ \\
\hline
\end{tabular}




\section{LITERATURE CITED}

Albrecht DR, Bargman Cl. 2011. High-content behavioral analysis of Caenorhabditis elegans in precise spatiotemporal chemical environments. Nat Methods 8:599-605.

Benzer S. 1967. Behavioral mutants of Drosophila isolated by countercurrent distribution. Proc Natl Acad Sci 58:1112-1119.

Bilen J, Bonini NM. 2005. Drosophila as a model for human neurodegenerative disease. Annu Rev Genet 39:153-171.

Branson K, Robie AA, Bender J, Perona P, Dickinson MH. 2009. Highthroughput ethomics in large groups of Drosophila. Nat Methods 6:451-457.

Connolly K. 1967. Locomotor activity in Drosophila III. A distinction between activity and reactivity. Anim Behav 15:149-152.

Cox PA, Banack SA, Murch SJ. 2003. Biomagnification of cyanobacterial neurotoxins and neurodegenerative disease among the Chamorro people of Guam. Proc Natl Acad Sci 110:13380-13383.

Dankert H, Wang L, Hoopfer ED, Anderson DJ, Perona P. 2009. Automated monitoring and analysis of social behavior in Drosophila. Nat Methods 6:297-303.

Feany MB, Bender WW. 2000. A Drosophila model of Parkinson's disease. Nature 404:394-398.

Featherstone DE, Rushton E, Rohrbough J, Liebl F, Karr J, Q. Sheng Q, Rodesch CK, Broadie K. 2005. An essential Drosophila glutamate receptor subunit that functions in both central neuropil and neuromuscular junction. J Neurosci 25:3199-3208.

Fry SN, Sayaman R, Dickinson MH. 2003. The aerodynamics of freeflight maneuvers in Drosophila. Science 300:495-498.

Gargano JW, Martin I, Bhandarib P, Grotewiel MS. 2005. Rapid iterative negative geotaxis (RING): a new method for assessing agerelated locomotor decline in Drosophila. Exp Gerontol 40:386-395.

Giacomotto J, Ségalat L. 2010. High-throughput screening and small animal models, where are we? Br J Pharmacol 160:204-216.

Götz KG. 1980. Visual guidance in Drosophila. In: Siddiqi O, Babu P, Hall LM, Hall JC, editors. Basic life sciences: development and neurobiology of Drosophila. New York: Plenum Press. Vol. 16 p 391-407.

Greene JC, Whitworth AJ, Kuo I, Andrews LA, Feany MB, Pallanck LJ. 2003. Mitochondrial pathology and apoptotic muscle degeneration in Drosophila parkin mutants. Proc Natl Acad Sci 100:4078-4083. 
Grover D, Tower J, Tavare S. 2008. O fly, where art thou? J R Soc Interface 5:1181-119.

Harper DG, Blake RW. 1989. Critical analysis of the use of highspeed film to determine maximum accelerations of fish. J Exp Biol 142:465-471.

Hirsch J. 1959. Studies in experimental behavior genetics. II. Individual differences in geotaxis as a function of chromosome variations in synthesized Drosophila populations. J Comp Physiol Psychol 52(3):304-308.

Hirsch J, Erlenmeyer-Kimling L. 1962. Studies in experimental behavior genetics. IV. Chromosome analyses for geotaxis. J Comp Physiol Psychol 55:732-739.

Hostetter RC, Hirsch J. 1967. Genetic analysis of geotaxis in Drosophila melanogaster: complementation between forward and reverse selection lines. J Comp Physiol Psychol 63:6670.

Hoy JB, Koehler PG, Patterson RS. 1996. A microcomputer-based system for real-time analysis of animal movements. J Neurosc Methods 64:157-161.

lijima K, Liu H-P, Chiang A-S, Hearn SA, Konsolaki M, Zhong Y. 2004. Dissecting the pathological effects of human Abeta40 and Abeta42 in Drosophila: a potential model for Alzheimer's disease. Proc Natl Acad Sci 101:6623-6628.

Johnston DS. 2002. The art and design of genetic screens: Drosophila melanogaster. Nat Rev 3:176-188.

Karamyan VT, Speth RC. 2008. Animal models of BMAA neurotoxicity: a critical review. Life Sci 82:233-246.

Karlsson O, Roman E, Berg A-L, Brittebo EB. 2011. Early hippocampal cell death, and late learning and memory deficits in rats exposed to the environmental toxin BMAA (B-Nmethylamino-I-alanine) during the neonatal period. Behav Brain Res 219:310-320.

Katsov AY, Clandinin TR. 2008. Motion processing streams in Drosophila are behaviorally specialized. Neuron 59:322-335.

LeBourg, E. 1983. Patterns of movement and ageing in Drosophila melanogaster. Arch Gerontol Geriatr 2:299-306.

Lessing D, Bonini NM. 2009. Maintaining the brain: insight into human neurodegeneration from Drosophila melanogaster mutants. Nat Rev Genet 10:359-370.

Lima SQ, Miesenböck G. 2005. Remote control of behavior through genetically targeted photostimulation of neurons. Cell 121:141- 152. 
Ling D, Song H-J, Garza D, Neufeld TP, Salvaterra PM. 2009. Abeta42-induced neurodegeneration via an age-dependent autophagic-lysomal injury in Drosophila. PLoS One 4(1):e4201. doi:10.1371/journal.pone.0004201.

Markow TA, Merriam J. 1977. Phototactic and geotactic behavior of counter-current defective mutants of Drosophila melanogaster. Behav Genet 7:447-455.

Martin JR. 2003. Locomotor activity: a complex behavioral trait to unravel. Behav Process 64:145-160.

Martin JR. 2004. A Portrait of locomotor behavior in Drosophila determined by video-tracking paradigm. Behav Process 67: 207-219.

Martin JR, Ernst R, Heisenberg M. 1999a. Temporal pattern of locomotor activity in Drosophila melanogaster. J Comp Physiol A 84:73-84.

Martin JR, Raabe T, Heisenberg M. 1999b. Central complex substructures are required for the maintenance of locomotor activity in Drosophila melanogaster. J Comp Physiol A 185:277- 288.

Pandey UB, Nichols CD. 2011. Human disease models in Drosophila melanogaster and the role of the fly in therapeutic drug discovery. Pharm Rev 63:411-436.

Peterson RT, Nass R, Boyd WA, Freedman JH, Dong K, Narahashi T. 2008. Use of nonmammalian alternative models for neurotoxicological study. NeurToxicology 29:546-555.

Pienaar IS, Götz J, Feany MB. 2010. Parkinson's disease: insights from non-traditional model organisms. Prog Neurobiol 92:558-571.

Rand MD. 2010. Drosophotoxicology: the growing potential for Drosophila in neurotoxicology. Neurotoxol Teratol. 32:74-83.

Rao SD, Banack SA, Cox PA, Weiss JH. 2006. BMAA selectively injures motor neurons via AMPA/kainate receptor activation. Exp Neurol 201:244-252.

Ricker JP, Hirsch J. 1988. Genetic changes occurring over 500 generations in lines of Drosophila melanogaster selected divergently for geotaxis. Behav Genet 18:13-25.

Ristroph L, Berman GJ, Bergou AJ,Wang ZJ, Cohen I. 2009. Automated hull reconstruction motion tracking (HRMT) applied to sideways maneuvers of free-flying insects. J Exp Biol 212:1324-1335.

Ruan H,WuC-F. 2008. Social interaction-mediated lifespan extension of Drosophila Cu/Zn superoxide dismutase mutants. Proc Natl Acad Sci 105:7506-7510.

Simon JC, Dickinson MH. 2010. A new chamber for studying the behavior of Drosophila. PLoS ONE 5(1):e8793. doi:10.1371/journal.pone.0008793. 
Slawson JB, Kim EZ, Griffith LC. 2009. High-resolution video tracking of locomotion in adult Drosophila melanogaster. J Visual Exp 24:doi:10.3791/1096.

Strauss R. 2002. The central complex and the genetic dissection of locomotor behavior. Curr Opin in Neurobiol 12: 633-638.

Strauss R, Heisenberg M. 1993. A higher control center of locomotor behavior in the Drosophila brain. J Neurosci 13:1852-1861.

Tiwari AK, Pragya P, Ram KR, Chowdhuri DK. 2011. Environmental chemical mediated male reproductive toxicity: Drosophila melanogaster as an alternative model. Theriogenology 76:197- 216 .

Toma DP, White KP, Hirsch J, Greenspan RJ. 2002. Identification of genes involved in Drosophila melanogaster geotaxis, a complex behavioral trait. Nat Genet 31:349-353.

Usherwood PNR. 1994. Insect glutamate receptors. Adv Insect Physiol 24:309-341.

Valente D, Golani I, Mitra PP. 2007. Analysis of the trajectory of Drosophila melanogaster in a circular open field arena. PLoS ONE 2(10):e1083. doi:10.1371/journal.pone.0001083.

Walker JA. 1998. Estimating velocities and accelerations of animal motions: a simulation experiment comparing numerical differentiation algorithms. J Exp Biol 201:981-995.

Wehrhahn C, Poggio T, Bülthoff H. 1982. Tracking and chasing in houseflies (Musca). Biol Cybern 45:123-130.

Weiss JH, Christine CW, Choi DW. 1989. Bicarbonate dependence of glutamate receptor activation by ß-N-methylamino-L-alanine: channel recording and study with related compounds. Neuron 3:321-326.

Whiting MG. 1988. Toxicity of cycads: implications for neurodegenerative diseases and cancer. In: Transcripts of four cycad conferences. The First Cycad Conference: 1962. New York: Third World Medical Research Foundation. p 1-17.

Wolf FW, Rodan AR, Tsai LT, Heberlein U. 2002. High-resolution analysis of ethanol-induced locomotor stimulation in Drosophila. J Neurosci 22:11035-11044.

Zhou X, Escala W, Papapetropoulos S, Bradley WG, Zhai RC. 2009. BMAA neurotoxicity in Drosophila. Amyotroph Lateral Scler 10:61-66.

Zhou X, Escala W, Papapetropoulos S, Zhai RC. 2010. ß-Nmethylamino- L-alanine induces neurological deficits and shortened life span in Drosophila. Toxins 2:2663-2679. 